\title{
Neighbour-distinguishing edge colourings of random regular graphs
}

\author{
Catherine Greenhill * \\ School of Mathematics and Statistics \\ University of New South Wales \\ Sydney NSW 2052, Australia \\ csg@unsw.edu.au
}

\author{
Andrzej Ruciński \\ Department of Discrete Mathematics \\ Adam Mickiewicz University \\ Poznań, Poland \\ rucinski@amu.edu.pl
}

Submitted: Dec 26, 2005; Accepted: Aug 10, 2006; Published: Aug 25, 2006

\begin{abstract}
A proper edge colouring of a graph is neighbour-distinguishing if for all pairs of adjacent vertices $v, w$ the set of colours appearing on the edges incident with $v$ is not equal to the set of colours appearing on the edges incident with $w$. Let $\operatorname{ndi}(G)$ be the least number of colours required for a proper neighbour-distinguishing edge colouring of $G$. We prove that for $d \geq 4$, a random $d$-regular graph $G$ on $n$ vertices asymptotically almost surely satisfies $\operatorname{ndi}(G) \leq\lceil 3 d / 2\rceil$. This verifies a conjecture of Zhang, Liu and Wang for almost all 4-regular graphs.
\end{abstract}

\section{Introduction}

Suppose that $G=(V, E)$ is a graph and $h: E \rightarrow[k]$ is a proper edge colouring of $G$. All edge colourings considered in this paper are proper and from now on we will not explicitly mention this. For each vertex $v \in V$, let $S(v)=\{h(e): v \in e\}$ be the set of colours on the neighbourhood of $v$. An edge colouring $h$ is said to be neighbour-distinguishing if $S(v) \neq S(w)$ for all $\{v, w\} \in E$. A neighbour-distinguishing edge colouring of $G$ exists if $G$ has no isolated edges. Let the neighbour-distinguishing index of $G$, denoted by $\operatorname{ndi}(G)$, be the least number of colours needed in a neighbour-distinguishing edge colouring of $G$ (where $\operatorname{ndi}(G)=\infty$ if $G$ contains an isolated edge). We sometimes abbreviate "neighbourdistinguishing edge colouring" to "nd-colouring". This notion was introduced by Zhang, Liu and Wang in [12]. (Note that nd-colourings are also called strong edge colourings [12] or adjacent vertex distinguishing colourings [2]. Our terminology and notation follows [4].)

\footnotetext{
*Research supported by the UNSW Faculty Research Grants Scheme.
} 
As an example which will be important in our proof, the cycle $C_{n}$ of length $n \geq 3$ has

$$
\operatorname{ndi}\left(C_{n}\right)= \begin{cases}3 & \text { if } n \equiv 0(\bmod 3) \\ 4 & \text { if } n \neq 5 \text { and } n \neq \equiv(\bmod 3) \\ 5 & \text { if } n=5 .\end{cases}
$$

Let $\Delta(G)=\Delta$ be the maximum degree of the graph $G$. Clearly ndi $(G) \geq \Delta$, and if there are adjacent vertices of maximum degree in $G$ then $\operatorname{ndi}(G) \geq \Delta+1$. Zhang, Liu and Wang [12] conjectured that

$$
\operatorname{ndi}(G) \leq \Delta+2
$$

whenever $G$ is a connected graph with at least three vertices which is not $C_{5}$. Balister et al. [2] proved the conjecture for all graphs with $\Delta=3$, as well as for all bipartite graphs. They also showed that the bound is tight.

Only much weaker bounds are known for general graphs without isolated edges. Akbari et al. [1] obtained the bound

$$
\operatorname{ndi}(G) \leq 3 \Delta
$$

for all graphs $G$ without isolated edges. For very large $\Delta$, Hatami [7] improved that to

$$
\operatorname{ndi}(G) \leq \Delta+300
$$

(if $\Delta \geq 10^{20}$ ), and Ghandehari and Hatami [6] proved that

$$
\operatorname{ndi}(G) \leq \Delta+27 \sqrt{\Delta \log \Delta}
$$

(if $\Delta \geq 10^{6}$ ). For $k$-chromatic graphs $G$, Balister et al. [2] proved the bound

$$
\operatorname{ndi}(G)=\Delta+O(\log k)=\Delta+O(\log \Delta),
$$

with an implicit constant in the $O(\cdot)$ term (see Remark 1 below).

In related work, Baril, Kheddouci and Togni [3] proved that $\operatorname{ndi}(G)=\Delta+1$ whenever $G$ is a multidimensional mesh or a hypercube, and Edwards, Hornak and Wozniak [4] showed that $\operatorname{ndi}(G) \leq \Delta+1$ if $G$ is a planar bipartite graph with $\Delta \geq 12$.

The main goal of this note is to verify the above conjecture for almost all 4-regular graphs, and to establish bounds on $\operatorname{ndi}(G)$ for almost all $d$-regular graphs $G$, where $d \geq 4$ is constant. Let $\mathcal{G}_{n, d}$ be the uniform probability space of all $d$-regular graphs on vertex set $[n]=\{1,2, \ldots, n\}$, where $n d$ is even. Here $d$ is a fixed constant and our asymptotics are as $n$ tends to infinity. Following [9], we will identify the probability space $\mathcal{G}_{n, d}$ with a random graph sampled from it. The phrase asymptotically almost surely (a.a.s.) means "with probability which tends to 1 as $n$ tends to infinity".

Theorem 1 Let $d \geq 4$. Then a.a.s. $\operatorname{ndi}\left(\mathcal{G}_{n, d}\right) \leq\lceil 3 d / 2\rceil$.

We prove this theorem with the aid of contiguity. Section 2 contains background on contiguity of random regular graphs. The main proof is presented in Section 3, while two crucial probabilistic claims used in that proof are deferred to Section 4 . 
Remark 1 Clearly, for very large $d$, our bound is superceded by the above mentioned results from [2], [7] and [6]. (Note that, by Brooks' theorem, every connected, $d$-regular, $n$-vertex graph is $d$-chromatic for $d \geq 3$ and $n \geq d+2$.) But our bound beats the bound from [2] for $d \leq 56$. Indeed, it follows from the proof given in [2] that

$$
d-1+5\left\lceil\log _{2} d\right\rceil
$$

is a lower bound on the upper bound in (1), and it is easy to check that the inequality

$$
\lceil 3 d / 2\rceil<d-1+5\left\lceil\log _{2} d\right\rceil
$$

holds for $d \leq 56$.

\section{Contiguity background}

Two sequences of probability spaces $\mathcal{A}_{n}$ and $\mathcal{B}_{n}$, with the same underlying set $\Omega_{n}$, are said to be contiguous (written $\mathcal{A}_{n} \approx \mathcal{B}_{n}$ ) if for any sequence of events $\left(\mathcal{E}_{n}\right)$ with $\mathcal{E}_{n} \subseteq \Omega_{n}$ for $n \geq 1$, we have

$$
\mathbb{P}_{\mathcal{A}_{n}}\left(\mathcal{E}_{n}\right) \rightarrow 1 \text { if and only if } \mathbb{P}_{\mathcal{B}_{n}}\left(\mathcal{E}_{n}\right) \rightarrow 1 \text {. }
$$

That is, the same (sequences of) events hold a.a.s. in both sequences of probability spaces. See [8], [11] or [9, Chapter 9] for more information about contiguity.

\subsection{Random regular multigraphs}

Let $\mathcal{G}_{n, d}^{\prime}$ be the (non-uniform) probability space of all $d$-regular multigraphs on vertex set $[n]$ with no loops, which arise from the pairings model (see [9, Chapter 9] or [11]). If $G$ is a $d$-regular multigraph on $[n]$ with no loops and with $r_{k}$ edges of multiplicity $k$, for $k \geq 1$, then the probability of $G$ in this model is proportional to $\prod_{k \geq 1}(k !)^{-r_{k}}$. In particular, the probability space obtained from $\mathcal{G}_{n, d}^{\prime}$ by conditioning on no multiple edges is exactly the space $\mathcal{G}_{n, d}$ of uniformly random $d$-regular (simple) graphs on $[n]$. (For readers unfamiliar with the pairings model, it does not hurt much to instead think of uniformly random $d$-regular multigraphs with no loops, since this model is contiguous with $\mathcal{G}_{n, d}^{\prime}$ (see $[8$, Theorem 12]).)

The definition of neighbour-distinguishing colourings and the neighbour-distinguishing index $\operatorname{ndi}(G)$ extend naturally to all multigraphs with no connected component of order two. (Define $\operatorname{ndi}(G)=\infty$ if $G$ has a connected component of order two.) For technical reasons, we will prove an analogue of Theorem 1 for the multigraph model $\mathcal{G}_{n, d}^{\prime}$. That is, we will prove the following.

Theorem 2 Let $d \geq 4$. Then a.a.s. $\operatorname{ndi}\left(\mathcal{G}_{n, d}^{\prime}\right) \leq\lceil 3 d / 2\rceil$.

Since the probability that $\mathcal{G}_{n, d}^{\prime}$ has no multiple edges is bounded away from 0 (see for example [8, Remark 13]), Theorem 1 follows immediately from Theorem 2. Indeed, for every event $\mathcal{E}_{n}$ which holds a.a.s. in $\mathcal{G}_{n, d}^{\prime}$, we have

$$
\mathbb{P}_{\mathcal{G}_{n, d}}\left(\neg \mathcal{E}_{n}\right)=\mathbb{P}_{\mathcal{G}_{n, d}^{\prime}}\left(\neg \mathcal{E}_{n} \mid \mathcal{G}_{n, d}^{\prime} \text { has no multiple edges }\right)=o(1) \text {. }
$$




\subsection{Contiguity arithmetic}

For given multigraphs $A$ and $B$ on the vertex set [n], the sum of $A$ and $B$, written $A+B$, is the multigraph on $[n]$ with edges given by the multiset union of the edges of $A$ and $B$. Define the sum of more than two multigraphs in the same way.

If $\mathcal{A}_{n}$ and $\mathcal{B}_{n}$ are both probability spaces on the set $\Omega_{n}$ of all multigraphs on the vertex set $[n]$, then their sum $\mathcal{A}_{n}+\mathcal{B}_{n}$ is the probability space obtained by choosing $A \in \mathcal{A}_{n}$ and $B \in \mathcal{B}_{n}$ independently and forming the multigraph $A+B$. Denote the sum of $k$ copies of $\mathcal{A}_{n}$ by $k \mathcal{A}_{n}$.

Now we list all contiguity instances which are relevant to our proof. Let $\mathcal{H}_{n}$ be the uniform probability space on the set of all Hamilton cycles on the vertex set $[n]$. Frieze et al. [5] proved that for $d \geq 3$,

$$
\mathcal{G}_{n, d}^{\prime} \approx \mathcal{G}_{n, d-2}^{\prime}+\mathcal{H}_{n}
$$

while Kim and Wormald [10] proved that

$$
\mathcal{G}_{n, 4}^{\prime} \approx 2 \mathcal{H}_{n} .
$$

The contiguous decompositions (2) and (3) give rise to an inductive proof of Theorem 2 , described in the next section.

\section{Proof of Theorem 2}

In this section we give the proof of Theorem 2, which was already shown to yield our main result, Theorem 1 . We begin with an outline of the proof.

\subsection{Outline of the proof}

We will prove Theorem 2 by induction on $d$, with increments of two (separately for $d$ odd and even), and with the inductive step based on the contiguous decomposition (2) and the following deterministic lemma.

Lemma 1 Fix $d \geq 5$ and $n \geq 6$. Let $G$ be $a(d-2)$-regular multigraph $G$ on the vertex set $[n]$ and let $H=C_{n}$ be a Hamilton cycle on the same vertex set $[n]$. Then

$$
\operatorname{ndi}(G+H) \leq \operatorname{ndi}(G)+3 .
$$

There are two base cases, namely $d=3$ and $d=4$. A result from [2] implies that $\operatorname{ndi}(G) \leq 5$ for all multigraphs $G$ with maximum degree 3 and no connected component of size 2 .

The following lemma provides the second base case.

Lemma 2 A.a.s. $\operatorname{ndi}\left(2 \mathcal{H}_{n}\right) \leq 6$. 
Let us see now how these two lemmas yield the proof of Theorem 2 .

Proof of Theorem 2. Note that when $d=3$ the contiguity result $(2)$ implies that $\mathcal{G}_{n, 3}^{\prime}$ is a.a.s. Hamiltonian, and hence connected. In particular, a.a.s. $\mathcal{G}_{n, 3}^{\prime}$ has no connected component of order two. Using this fact the theorem holds when $d=3$, by [2]. By Lemma 2 and (3), the theorem holds when $d=4$. Since

$$
\lceil 3(d-2) / 2\rceil+3=\lceil 3 d / 2\rceil
$$

the result follows by induction for all $d \geq 3$, using Lemma 1 and (2).

As an aside, note that working with graphs rather than multigraphs and substituting the deterministic upper bound of 8 for the asymptotically almost sure upper bound of 6 in Lemma 2 gives the following deterministic result.

Lemma 3 Let $G$ be a d-regular graph on the vertex set $[n]$.

(i) If $d$ is odd and the edge set of $G$ can be partitioned into the edge sets of $(d-3) / 2$ disjoint Hamilton cycles and one cubic graph then $\operatorname{ndi}(G) \leq\lceil 3 d / 2\rceil$.

(ii) If $d$ is even and the edge set of $G$ can be partitioned into the edge sets of $d / 2$ disjoint Hamilton cycles then $\operatorname{ndi}(G) \leq\lceil 3 d / 2\rceil+2$.

Now we continue with the proof of Theorem 2. It remains to prove Lemma 1 and Lemma 2. Both lemmas are quite trivial for $n \equiv 0(\bmod 3)$ while some difficulties arise in the other cases. We handle each value of $n(\bmod 3)$ separately.

In what follows, we say that vertices $v$ and $w$ are distinguishable under a given edge colouring if $S(v) \neq S(w)$. (Here $v$ and $w$ need not be neighbours.) Vertices which are not distinguishable will be called indistinguishable.

The following fact, though obvious, is quite useful in the proofs.

Fact 1 Let $G_{1}$ and $G_{2}$ be multigraphs on the same vertex set. Then

$$
\operatorname{ndi}\left(G_{1}+G_{2}\right) \leq \operatorname{ndi}\left(G_{1}\right)+\operatorname{ndi}\left(G_{2}\right) .
$$

Proof. The inequality holds trivially if either $G_{1}$ or $G_{2}$ has a component of size two. Suppose then that $G_{i}$ has an nd-colouring $h_{i}$ with the set of colours $C_{i}$ for $i=1,2$, where $C_{1} \cap C_{2}=\emptyset$. We define an edge colouring $h$ of $G_{1}+G_{2}$ using the colours in $C_{1} \cup C_{2}$ by letting $h(e)=h_{i}(e)$ if $e \in G_{i}, i=1,2$. It is easy to check that $h$ is an nd-colouring of $G_{1}+G_{2}$.

Note that for $n \not \equiv 0(\bmod 3)$ and $n \geq 6$ we have $\operatorname{ndi}\left(C_{n}\right)=4$. Thus Lemma 1 can be viewed as a sharpening (by 1 ) of Fact 1 when $G_{2}=C_{n}$. Moreover, Lemma 2 shows that in the special case when also $G_{1}=C_{n}$ we gain 2 a.a.s. if $G_{2}$ is drawn randomly from $\mathcal{H}_{n}$. The idea behind these improvements is to allow some pairs of vertices to be indistinguishable in the colouring of $G_{2}$, provided that they are distinguishable in the colouring of $G_{1}$. 


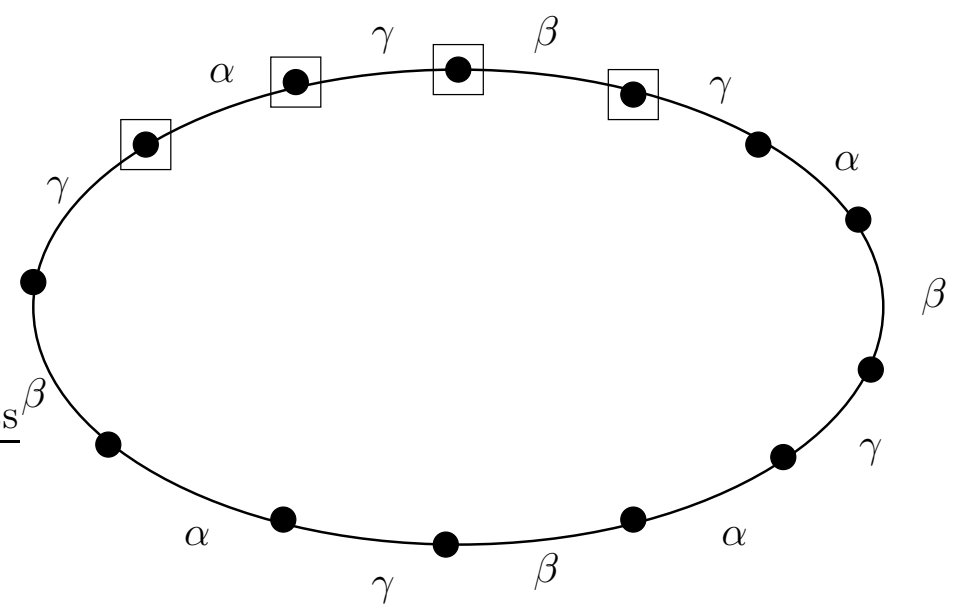

Figure 1: The colouring of $H$ used in the second case of the proof of Lemma 1 when $n \equiv 1$ $(\bmod 3)$

\subsection{Proof of Lemma 1}

Fix $d \geq 5, n \geq 6$, and let $G$ be a $(d-2)$-regular multigraph on the vertex set $[n]$. If $G$ has a connected component of size two then the lemma holds trivially, so we may assume that $G$ has no such component. If $n \equiv 0(\bmod 3)$ then $\operatorname{ndi}\left(C_{n}\right)=3$ and Lemma 1 holds (deterministically) by Fact 1. Otherwise, fix an optimal nd-colouring $h$ of $G$ and suppose that $h$ uses the colour set $[r]$. Let $H=C_{n}$ be a Hamilton cycle on the same vertex set $[n]$.

Case $n \equiv 1(\bmod 3)$ :

Suppose that there exists an edge $u v$ of $H$ such that some colour $\delta \in[r]$ is missing at both $u$ and $v$. Then we may colour $u v$ with the colour $\delta$ in $H$, and colour the rest of $H$ with three new colours to give an nd-colouring of $H$. This gives an nd-colouring of $G+H$ using $r+3$ colours.

On the other hand, if no such edge exists in $H$ then for every edge $u v$ of $H$ we have $|S(u) \cup S(v)|=r$. Since $G$ is $(d-2)$-regular we know that $r \geq d-1$, which implies that $|S(u) \cap S(v)| \leq d-3$. Thus there is at least one colour in $S(u)-S(v)$, which implies that $u$ and $v$ are distinguishable under $h$. As this holds for any edge of $H$, consider four consecutive vertices $u_{1}, \ldots, u_{4}$ of $H$. We may colour $H$ with three new colours in such a way that all vertices are distinguishable from their $H$-neighbours except for the pairs $u_{1}, u_{2}$ and $u_{3}, u_{4}$. (See Figure 1 for an example, where the vertices $u_{1}, \ldots, u_{4}$ have boxes drawn around them.) This gives an $n d$-colouring of $G+H$ using $r+3$ colours.

Case $n \equiv 2(\bmod 3)$ :

Let $V_{1}, \ldots, V_{k}$ be the partition of $[n]$ given by the colour classes of the (proper) vertex colouring of $G$ induced by $h$. That is, vertices $v$ and $w$ belong to the same part of the partition if and only if $S(v)=S(w)$ under $h$. (Here $k$ is the number of distinct sets $S(v)$ under $h$, which could be as large as $\left(\begin{array}{l}r \\ d\end{array}\right)$.) 


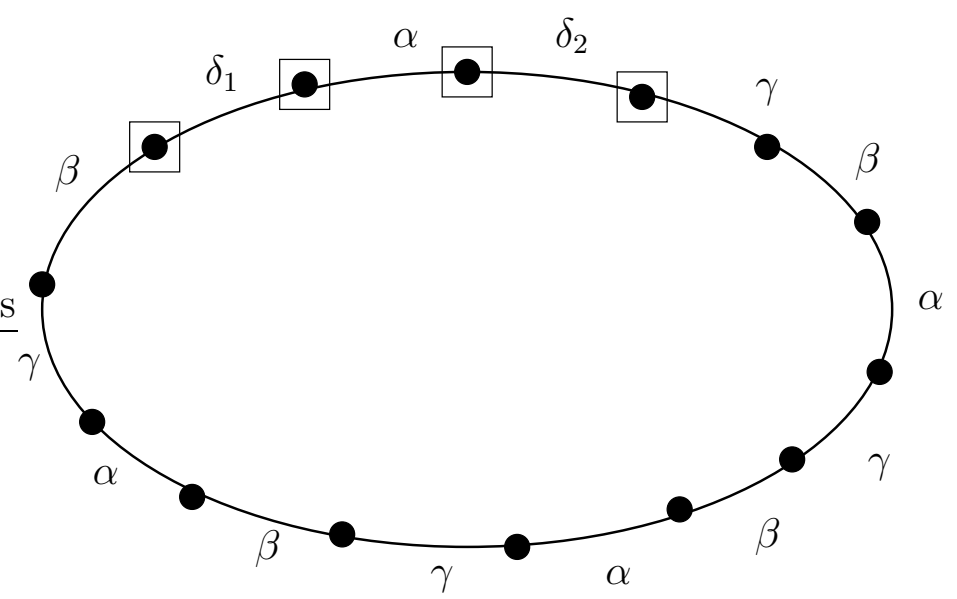

Figure 2: The colouring of $H$ used in the second case of the proof of Lemma 1 when $n \equiv 2$ $(\bmod 3)$

First suppose that there is a 2-path uvw on $H$ such that $u$ and $v$ are distinguishable under $h$ and $v$ and $w$ are distinguishable under $h$. Then we may colour the edges of $H$ using three new colours in such a way that every vertex is distinguishable from its $H$ neighbours except for the pairs $u, v$ and $v, w$. This gives an nd-colouring of $G+H$ using $r+3$ colours.

Next, suppose that there is no such 2-path on $H$. Then whenever $H$ enters a set $V_{i}$, it stays in $V_{i}$ for at least one more vertex (that is, $H\left[V_{i}\right]$ has no isolated vertices). Choose an edge $u_{2} v_{1}$ of $H$ with $u_{2} \in V_{i}$ and $v_{1} \in V_{j}$ for some $i \neq j$. Then we have a 3 -path $u_{1} u_{2} v_{1} v_{2}$ in $H$ such that $u_{1}, u_{2} \in V_{i}$ and $v_{1}, v_{2} \in V_{j}$. Hence there exists distinct colours $\delta_{1}, \delta_{2} \in[r]$ such that $\delta_{1}$ is missing at $u_{1}$ and at $u_{2}$ and $\delta_{2}$ is missing at $v_{1}$ and at $v_{2}$. We may now construct an nd-colouring of $H$ using $\delta_{1}$ for the edge $u_{1} u_{2}, \delta_{2}$ for the edge $v_{1} v_{2}$, and using three new colours for all other edges of $H$. (See Figure 2 for an example, where the vertices $u_{1}, u_{2}, v_{1}, v_{2}$ have boxes around them.) This produces an nd-colouring of $G+H$ using $r+3$ colours, as required, completing the proof of Lemma 1.

\subsection{Proof of Lemma 2}

Again, if $n \equiv 0(\bmod 3)$ then $\operatorname{ndi}\left(C_{n}\right)=3$ and Lemma 2 holds (deterministically) using Fact 1. Otherwise, write $G=H_{1}+H_{2}$, where $H_{1}$ and $H_{2}$ are two Hamilton cycles on $[n]$. Assume that $H_{1}$ is fixed and that $H_{2}$ is a random element of $\mathcal{H}_{n}$.

Case $n \equiv 1(\bmod 3)$ :

We will show in Claim 1 below (see Section 4$)$ that when $n \equiv 1(\bmod 3)$, a.a.s. there is an edge $v w$ of $H_{2}$ such that the distance from $v$ to $w$ in $H_{1}$ is congruent to $2(\bmod 3)$ (in which case both paths from $v$ to $w$ in $H_{1}$ have lengths congruent to $2(\bmod 3)$ ).

Colour the edge $v w$ with the colour $\gamma$, and colour the rest of $H_{2}$ with colours $\delta, \epsilon, \zeta$ to give an nd-colouring of $H_{2}$. Next, colour the edges of $H_{1}$ with colours $\alpha, \beta, \gamma$ in such a way that $v$ is adjacent to edges coloured $\alpha, \beta$ and so is $w$, and all adjacent vertices of $H_{2}$ 
are distinguishable except that $v$ and $w$ are not distinguishable from their neighbours. To achieve this, use the colouring

$$
\alpha, \beta, \gamma, \alpha, \beta, \gamma, \ldots, \alpha, \beta
$$

from $v$ to $w$ around one side of $H_{1}$, and use the colouring

$$
\beta, \alpha, \gamma, \beta, \alpha, \gamma, \ldots, \beta, \alpha
$$

from $v$ to $w$ around the other side (see Figure 3). In the induced edge-colouring of

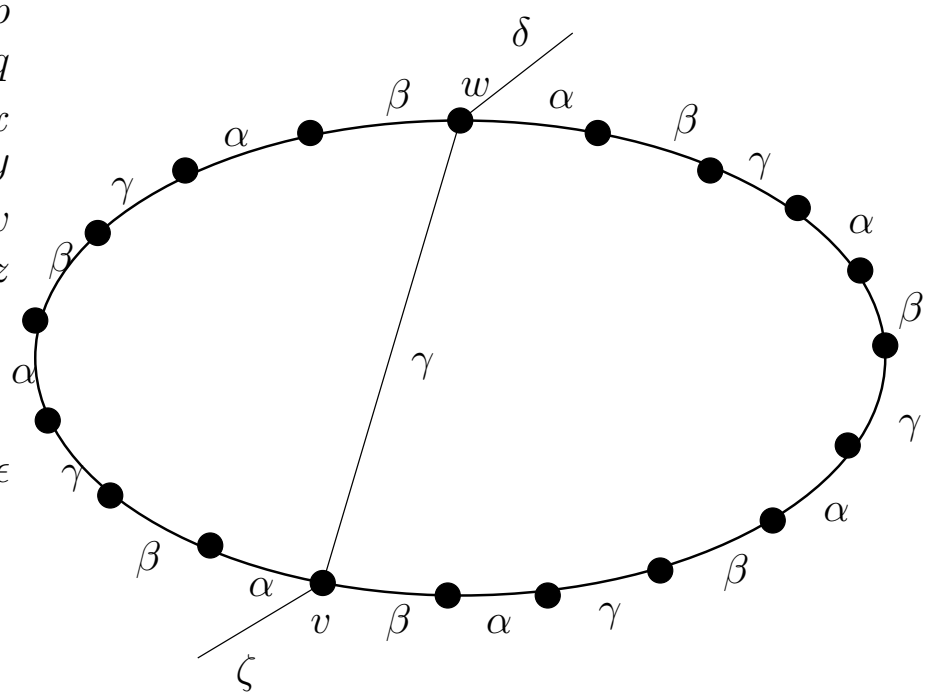

Figure 3: The colouring of $H_{1}$ used in the proof of Lemma 2 when $n \equiv 1(\bmod 3)$

$H_{1}+H_{2}$, vertices $v$ and $w$ are incident with three edges coloured with colours $\{\alpha, \beta, \gamma\}$, and they are the only two vertices in the multigraph with this property, which makes them distinguishable from their $H_{1}$-neighbours. So this is an nd-colouring of $H_{1}+H_{2}$.

Case $n \equiv 2(\bmod 3)$ :

We will show in Claim 2 below (see Section 4$)$ that when $n \equiv 2(\bmod 3)$, a.a.s. there exist edges $v_{1} w_{1}$ and $v_{2} w_{2}$ of $H_{2}$ which cut $H_{2}$ into two paths of positive lengths divisible by 3 , and such that the vertices $v_{1}, w_{1}, v_{2}, w_{2}$ cut $H_{1}$ into four paths, $P_{1}, \ldots, P_{4}$, of lengths congruent to $2(\bmod 3)$.

Colour $v_{1} w_{1}$ and $v_{2} w_{2}$ with colour $\gamma$ and colour the rest of $H_{2}$ by $\delta, \epsilon, \zeta$, so that all pairs of adjacent vertices are distinguishable. Finally, colour $H_{1}$ with colours $\alpha, \beta, \gamma$ so that each path $P_{i}$ begins and ends with the colour sequence $\alpha, \beta$ and all pairs of adjacent vertices on $H_{1}$ are distinguishable except that $v_{1}, w_{1}, v_{2}, w_{2}$ are not distinguishable from their neighbours on $H_{1}$. It follows similarly to the case when $n \equiv 1(\bmod 3)$ that all pairs of adjacent vertices of $H_{1}+H_{2}$ are distinguishable. 


\section{Adding a random Hamilton cycle}

It remains to prove the two final claims, both about the effect of adding a random Hamilton cycle to a given graph.

To choose a uniformly random Hamilton cycle $H$ on the set $[n]$, it will be convenient to consider the following random process. Take an arbitrary start-vertex $u_{1}$ and proceed randomly around $[n]$ creating $H$ vertex by vertex. Specifically, suppose that $u_{1} u_{2} \cdots u_{j}$ have already been chosen. Then $u_{j+1}$ is selected uniformly at random from the remaining $n-j$ vertices, for $j=1, \ldots, n-1$ (and the edge $u_{n} u_{1}$ is added at the end to complete the cycle). Every Hamilton cycle will have two chances to appear, one for each direction, each with probability $1 /(n-1)$ ! (and thus with global probability $2 /(n-1)$ !, as it should be). In this process, let $e_{i}=u_{i} u_{i+1}, i=1, \ldots, n$ be the $i$ th random edge of $H$. (The edge $e_{n}$ is not really random, since $u_{n+1}=u_{1}$.) Then, for each $i$ the sequence $\left(e_{1}, \ldots, e_{i}\right)$ will be called the history of $H$ until time $i$. We refer to this process and the notation described above throughout this section.

Throughout this section we will write $n / c$ instead of $\lfloor n / c\rfloor$ in a few places, where $c$ is a constant. Since $n$ tends to infinity the error in doing this is negligible.

Below, $H_{1}$ is a fixed Hamilton cycle on $[n]$, while $H_{2}$ is an element of $\mathcal{H}_{n}$ selected uniformly at random.

Claim 1 Suppose that $n \equiv 1(\bmod 3)$. Then a.a.s. $H_{2}$ contains an edge vw such that the distance from $v$ to $w$ in $H_{1}$ is congruent to $2(\bmod 3)$.

Proof. Choose $H_{2}$ vertex by vertex, as described above. Call the $i$ th edge $e_{i}=u_{i} u_{i+1}$ of $H_{2}$ bad if the distance from $u_{i}$ to $u_{i+1}$ in $H_{1}$ is not equal to $2(\bmod 3)$ (in some direction). Let $E_{i}$ be the event that $e_{i}$ is bad. Then

$$
\mathbb{P}\left(\bigcap_{i=1}^{n} E_{i}\right) \leq \mathbb{P}\left(\bigcap_{i=1}^{n / 12} E_{i}\right)=\prod_{i=1}^{n / 12} \mathbb{P}\left(E_{i} \mid \bigcap_{j=1}^{i-1} E_{j}\right)
$$

In order to estimate $\mathbb{P}\left(E_{i} \mid \bigcap_{j=1}^{i-1} E_{j}\right)$, we first estimate $\mathbb{P}\left(E_{i} \mid e_{1}, \ldots, e_{i-1}\right)$; that is, the probability of the event $E_{i}$ conditioned on the history of the process up to time $i$. Given $u_{i}$ there are at most $2 n / 3$ vertices which are not on $H_{2}$ yet, and which make a bad pair with $u_{i}$. Since we choose $u_{i+1}$ out of at least $n-n / 12=11 n / 12$ vertices, we have

$$
\mathbb{P}\left(E_{i} \mid e_{1}, \ldots, e_{i-1}\right) \leq 8 / 11
$$

Summing over all possible histories $e_{1}, \ldots, e_{i-1}$ such that $E_{1}, \ldots, E_{i-1}$ all hold, we obtain

$$
\mathbb{P}\left(E_{i} \mid \bigcap_{j=1}^{i-1} E_{j}\right) \leq 8 / 11
$$


Therefore

$$
\mathbb{P}\left(\bigcap_{i=1}^{n} E_{i}\right) \leq(8 / 11)^{n / 12}=o(1)
$$

as required.

Claim 2 Suppose that $n \equiv 2(\bmod 3)$. Then a.a.s. $H_{2}$ contains edges $v_{1} w_{1}$ and $v_{2} w_{2}$ which cut $\mathrm{H}_{2}$ into two paths of positive lengths divisible by 3 and such that the vertices $v_{1}, w_{1}, v_{2}, w_{2}$ cut $H_{1}$ into four paths, $P_{1}, \ldots, P_{4}$, of lengths congruent to $2(\bmod 3)$.

Proof. Call the edge $e_{i}=u_{i} u_{i+1}$ of $H_{2}$ good if the distance from $u_{i}$ to $u_{i+1}$ in $H_{1}$ is at most $n / 4$ and is congruent to $2(\bmod 3)$. We modify the proof of Claim 1 to show that a.a.s. there exists a good edge $e_{i}$ with $1 \leq i \leq n / 12$. Let $E_{i}$ be the event that edge $e_{i}$ is bad. Given the history up until step $i$, there are at most $n / 2$ choices for $u_{i+1}$ which (do not yet lie on $H_{2}$ and) are too far away from $u_{i}$ and at most $n / 3$ choices which (do not yet lie on $H_{2}$ and) are close enough to $u_{i}$ but with the wrong modulus. At least $11 n / 12$ vertices do not yet lie on $H_{2}$, so arguing as in Claim 1,

$$
\mathbb{P}\left(E_{i} \mid \bigcap_{j=1}^{i-1} E_{j}\right) \leq \frac{n / 2+n / 3}{11 n / 12}=10 / 11 .
$$

Therefore

$$
\mathbb{P}\left(\bigcap_{i=1}^{n / 12} E_{i}\right) \leq(10 / 11)^{n / 12}=o(1) .
$$

This says that a.a.s. there exists a good edge $e_{i}$ with $1 \leq i \leq n / 12$. This edge $e_{i}$ is the edge $v_{1} w_{1}$. Call this Phase 1 .

Assume for the rest of the proof that Phase 1 is successful (that is, a good edge was found in the first $n / 12$ steps). The vertices $v_{1}, w_{1}$ split $H_{1}$ into a short path (of length at most $n / 4)$ and a long path. Call the vertices of the long path active, and call the vertices of the short path inactive. In Phase 2, we say that the edge $e_{j}=u_{j} u_{j+1}$ is good if

(i) $u_{j}$ is active,

(ii) the distance from $u_{j}$ to the closer of $v_{1}, w_{1}$ in $H_{1}$ is at most $n / 4$ and is congruent to $2(\bmod 3)$,

(iii) $u_{j+1}$ is active,

(iv) the path from $u_{j}$ to $u_{j+1}$ in $H_{1}$ which does not contain $v_{1}, w_{1}$ has length congruent to $2(\bmod 3)$,

(v) if $P$ is the path in $H_{1}$ of length at most $n / 4$ between $u_{j}$ and the closer of $v_{1}, w_{1}$, then $u_{j+1}$ does not lie on $P$. 
We say that Phase 2 is successful if there exists a good edge $e_{j}$ such that $j=i+1+3 \ell$ where $1 \leq \ell \leq n / 72$. We will show that a.a.s. Phase 2 is successful, conditioned on Phase 1 being successful. If Phase 2 is sucessful then the edge $e_{j}$ is the edge $v_{2} w_{2}$.

For example, consider Figure 4. The edge $v_{1} w_{1}$ is shown, together with the possible choices for $u_{j}$ which satisfy (i) and (ii). Then for a particular choice of $u_{j}$, Figure 5 shows

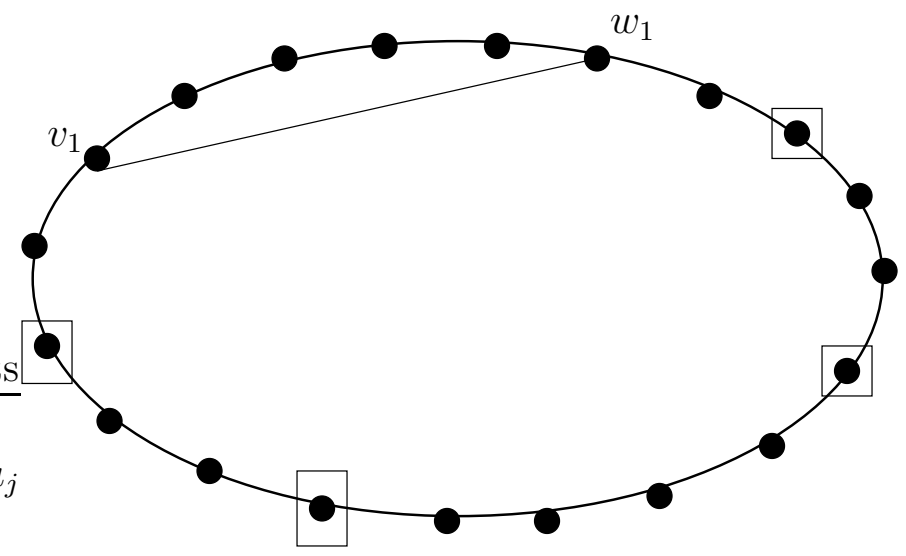

Figure 4: Choices for $u_{j}$ in Phase 2

the possible choices for $u_{j+1}$ which satisfy (iii)-(v).

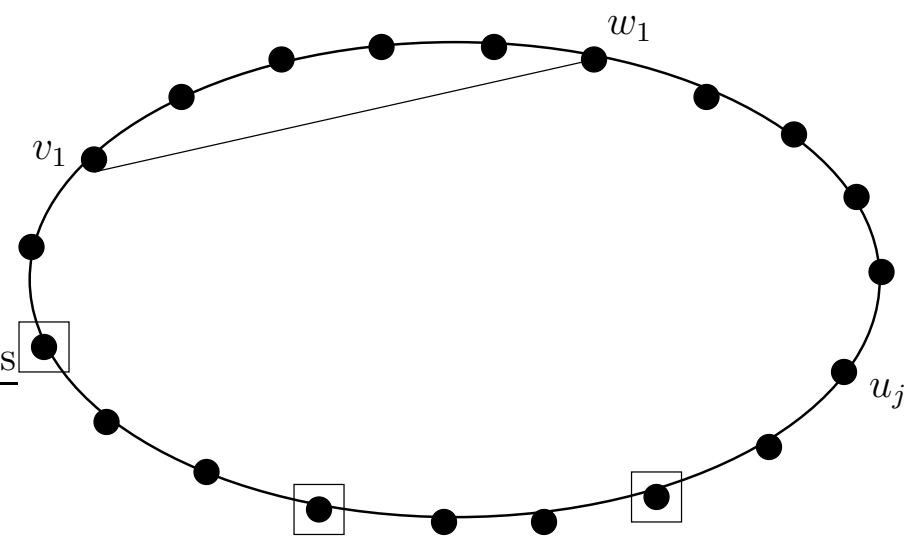

Figure 5: Choices for $u_{j+1}$ in Phase 2

Let $F_{j}$ be the event that $e_{j}$ is bad, where $j=i+1+3 \ell$ and $1 \leq \ell \leq n / 72$. Let $e_{1}, \ldots, e_{j-2}$ be the history up until step $j-1$, and assume that Phase 1 succeeds for this history. We next choose $u_{j}$, and this choice succeeds if (i) and (ii) hold. There are $n / 2$ active vertices which are close enough to $v_{1}$ or $w_{1}$, and $1 / 3$ of these have distance which is the correct modulus. Of these, at most $n / 8$ already lie on $H_{2}$. Therefore the probability that $u_{j}$ satisfies (i) and (ii), conditioned on the history up until step $j-1$, is at least $1 / 24$. If $u_{j}$ satisfies (i) and (ii) then the probability that $u_{j+1}$ satisfies (iii) - (v) is also at least $1 / 24$, since there are at least $n / 2$ active vertices which do not lie in $P$, of which $1 / 3$ of 
these have distance which is the correct modulus (in (iv)), and only at most $n / 8$ of these already lie on $\mathrm{H}_{2}$. It follows that

$$
\mathbb{P}\left(F_{j} \mid e_{1}, \ldots, e_{j-2}\right) \leq \frac{575}{576}
$$

and by the usual arguments, the probability that Phase 2 fails, conditioned on Phase 1 succeeding, is at most $(575 / 576)^{n / 72}=o(1)$. Hence a.a.s. Phases 1 and 2 both succeed, as required.

Acknowledgments: We are greatly indebted to Michał Karoński for drawing our attention to the problem of neighbour-distinguishing colourings. We would like to dedicate this paper to his sixtieth birthday.

\section{References}

[1] S. Akbari, H. Bidkhori and N. Nosrati, $r$-strong edge colorings of graphs, Discrete Mathematics (to appear).

[2] P.N. Balister, E. Györi, J. Lehel and R.H. Schelp, Adjacent vertex distinguishing edge-colorings, preprint (2002).

[3] J.-L. Baril, H. Kheddouci and O. Togni, Adjacent vertex distinguishing edge-colorings of meshes and hypercubes, Australasian Journal of Combinatorics 35 (2006), 89-102.

[4] K. Edwards, M. Hornak and M. Wozniak, On the neighbour-distinguishing index of a graph, preprint (2005).

[5] A. Frieze, M. Jerrum, M. Molloy, R. Robinson and N.C. Wormald, Generating and counting Hamilton cycles in random regular graphs, Journal of Algorithms 21 (1996), $176-198$.

[6] M. Ghandehari and H. Hatami, Two upper bounds for the strong edge chromatic number, preprint (2004).

[7] H. Hatami, $\Delta+300$ is a bound on the adjacent vertex distinguishing edge chromatic number, Journal of Combinatorial Theory (Series B) 95 (2005), 246-256.

[8] S. Janson, Random regular graphs: asymptotic distributions and contiguity, Combinatorics, Probability and Computing 4 (1995), pp. 369-405.

[9] S. Janson, T. Łuczak and A. Ruciński, Random Graphs, Wiley, New York, 2000.

[10] J.H. Kim and N.C. Wormald, Random matchings which induce Hamilton cycles, and hamiltonian decompositions of random regular graphs, Journal of Combinatorial Theory (Series B) $\mathbf{8 1}$ (2001), 20-44.

[11] N.C. Wormald, Models of random regular graphs, in Surveys in Combinatorics 1999 (J.D. Lamb and D.A. Preece, eds.), vol. 267 of LMS Lecture Note Series, Cambridge University Press, Cambridge, 1999, pp. 239-298.

[12] Z. Zhang, L. Liu and J. Wang, Adjacent strong edge coloring of graphs, Applied Mathematics Letters 15 (2002), 623-626. 